\title{
A Novel Approach to Design Chitosan-Polyester Materials for Biomedical Applications
}

\author{
Tatiana A. Akopova, ${ }^{1}$ Tatiana S. Demina, ${ }^{1}$ Alexander N. Shchegolikhin, ${ }^{2}$ \\ Tikhon S. Kurkin, ${ }^{1}$ Christian Grandfils, ${ }^{3}$ Nikolay S. Perov, ${ }^{1}$ \\ Alexander S. Kechekyan, ${ }^{1}$ and Alexander N. Zelenetskii ${ }^{1}$ \\ ${ }^{1}$ N. S. Enikolopov Institute of Synthetic Polymer Materials, Russian Academy of Sciences, 70 Profsoyuznaya Street, \\ Moscow 117393, Russia \\ ${ }^{2}$ N. M. Emanuel Institute of Biochemical Physics, Russian Academy of Sciences, 4 Kosygina Street, Moscow 119334, Russia \\ ${ }^{3}$ Centre Interfacultaire des Biomatériaux, University of Liege, B6c, 4000 Liège, Belgium
}

Correspondence should be addressed to Tatiana A. Akopova, akopova@ispm.ru

Received 26 November 2011; Accepted 12 March 2012

Academic Editor: Kibret Mequanint

Copyright (C) 2012 Tatiana A. Akopova et al. This is an open access article distributed under the Creative Commons Attribution License, which permits unrestricted use, distribution, and reproduction in any medium, provided the original work is properly cited.

A novel approach to design chitosan-polyester materials is reported. The method is based on mechanical activation and effective intermixing of the substrates under high pressure and shear deformation in the course of solid-state reactive blending. The marked departure of this approach from previous practice resides on exploitation of a variety of chemical transformations of the solid polymers that become feasible under conditions of plastic flow. Low temperatures (above $T_{g}$ but below the melting points of the crystalline polymers) are maintained throughout the process, minimizing mechanical and oxidative degradation of the polymers. Morphology as well as structural, mechanical, and relaxation properties of those prepared blends of chitosan with semicrystalline poly(L,L-lactide) and amorphous poly(D,L-lactide-co-glycolide) has been studied. Grafting of polyester moieties onto chitosan chains was found to occur under employed pressures and shear stresses. The prepared polymer blends have demonstrated an amphiphilic behavior with a propensity to disperse in organic solvents that widens possibilities to transform them into promising materials for various biomedical applications.

\section{Introduction}

Composites and graft copolymers of polysaccharides with polyesters, like chitosan and poly(lactide)s, are of great practical and theoretical interest. These hybrid materials synergistically combine functionalities of the polymers comprising them. Also they often possess new properties unusual for the parent macromolecules. Poly(L-lactide) (PLLA), a semicrystalline polymer with the melting temperature of $170-190^{\circ} \mathrm{C}$ and the glass transition temperature of $55-60^{\circ} \mathrm{C}$ [1], is attractive for biomedical applications as pins, plates, screws, intrabone and soft-tissue implants, and as vectors for sustained release of bioactive compounds due to its good mechanical properties, biocompatibility, and biodegradability [2]. Polylactide copolymers with poly(glycolic acid) (PLGA) possess an enhanced rate of degradation owing to disturbing of polyester crystallinity that allows to extend its application in tissue engineering [3]. However, a lack of the functional groups to promote cell attachment limits usage of polyesters in some biomedical fields, in particular for tissue engineering. Their ability to form compatible blends with some other polymers can provide an improvement of physical and biochemical properties of biomaterials based on PLLA and its copolymers [4].

Of particular interest is the blending with chitosan which is a deacetylated form of natural biopolymer chitin. Being composed mainly of 2 -amino-2-deoxy- $\beta$-D-glucopyranose (D-glucosamine) units, chitosan is considered to be most abundant polysaccharide for biomedical applications. The unique structure and properties of this natural cationic polymer make it valuable starting material for the synthesis of graft copolymers. Chitosan and its derivatives show 
excellent biocompatibility, biodegradability, low immunogenicity, and rather good biological activity $[5,6]$. The use of chitosan as a component of cartilage tissue scaffolds seems to be a promising approach to enhance chondrogenesis [7]. Recently, chitosan has been rendered as a promising biomaterial for vascular surgery, tissue culture, and tissue repair as well as a hemostatic agent $[8,9]$. A number of polymeric materials based on chitosan and poly(lactide)s in the form of films, porous scaffolds, micro- and nanoparticles have been synthesized and studied recently in terms of their use for tissue engineering and for controlled drug delivery [1012]. Also of importance, cytocompatibility of chitosan-gpoly(lactide) copolymer materials has been proven [13].

The well-known low compatibility of synthetic polymers with natural ones as well as unsuitability of polysaccharides for processing in the molten state strongly hinders the development and manufacture of many useful polysaccharidebased polymeric systems. Nonetheless, the reactive blending with thermoplastic polymers through the melting of the latter is rather often used in attempts to obtain blends of chitosan with aliphatic polyesters. High viscosity of the melts, being especially troublesome in fabrication of composite materials with high-chitosan content, normally requires adding glycerol and water as plasticizers. It results in changing adhesion for the worse as well as leads to hydrolysis of polyester matrix. The yield of graft copolymers facilitating the components compatibilization under such melt processing is insubstantial. The grafting occurs on the very surface of the polysaccharide particles. The size of chitosan particles remains practically unchanged as compared with the initial ones. The studies of morphology of these blends confirmed that chitosan and polyester are phase-separated systems [14]. Therefore, the growing needs of medicine and biotechnology call for innovative methods to design chitosan-polyester materials with desirable properties.

A promising method of the solid-state grafting of different synthetic polymers to infusible and poorly soluble polysaccharides (such as chitin and chitosan) was recently developed by the authors [15]. This approach hinges on activation of the substrate and the reactant during their intensive intermixing by applying external mechanical energy. The method has numerous advantages because the entire modification process proceeds in the solid state and does not require the components melting or employment of any solvents as the reaction medium. Under the joint action of high pressure and shear deformations, the solid material is shown to transit into the state of plastic flow [16]. The processing of polymers under such conditions is frequently able to induce substantial changes in their morphology and, in particular, leads to lowering the crystallinity, facilitates the particulates dispersability, and so forth $[17,18]$. The grinding of the substances under shear deformation, along with intermixing at the molecular level, leads to generation of structural defects, free radicals, double electric layers, and excited electron states [19]. The power of this technique relies upon a variety of chemical transformations (e.g. grafting, polymer-analogous, and cross-coupling reactions) made possible at the shear-induced plastic flow conditions. It is worth to note that, under these conditions, the reactions often proceed "spontaneously", that is, there is no need in introducing any initiators or catalysts.

The aim of this work is to study the morphology as well as structural, mechanical, and relaxation properties of the chitosan-polyester materials prepared through the solid-state reactive blending (SSRB) technique. Possibilities to design biodegradable scaffolds based on the obtained systems for their further application as matrices in tissue engineering are evaluated as well.

\section{Materials and Methods}

2.1. Materials. Commercially available poly(L-lactide) (PLLA) (Sigma) with an average $M_{w}$ of $160 \mathrm{kDa}$ and poly(D, L-lactide-co-glycolide) (PLGA) (Resomer, type 50:50, Boehringer Ingelheim) with $M_{w}$ of $52 \mathrm{kDa}$ were used throughout this study as received. Chitosan $\left(M_{w}=60 \mathrm{kDa}\right.$; acetylation degree of 0.10 ) has been prepared by the solidstate synthesis in ISPM RAS as reported earlier [20]. All solvents were purchased from Acros Organics as analytical grade and were used without further purification.

2.2. Processing of the Blends by SSRB Procedure. The chitosan-polyester blends were processed in a semi-industrial corotating twin-screw extruder (ZE 40A $\times$ 40D UTS, Berstorff, Germany) specially designed for powerful dispersion of solids. Before the final processing, chitosan powders were first premixed with polyester pellets by passing the weighted amounts of the components once through the extruder at $50^{\circ} \mathrm{C}$ and the screw rotation speed of $100 \mathrm{rpm}$. In this way, a series of $300 \mathrm{~g}$ batches of Chitosan/PLLA (CPL) mixtures characterized by ratio of the components of $40 / 60$ (w/w, CPL1) and 60/40 (w/w, CPL2) and Chitosan/PLGA (CPLG) mixtures of $60 / 40(\mathrm{w} / \mathrm{w})$ were prepared.

Final processing of the experimental blends characterized by the predetermined ratios of the components was performed at different discrete temperatures chosen within the range of $50-150^{\circ} \mathrm{C}$, that is, below the melting point of PLLA. Specifically, each premixed batch $(300 \mathrm{~g})$ was processed in the extruder for $10 \mathrm{~min}$ at the temperature of $50,60,100$, 110,130 or $150^{\circ} \mathrm{C}$, in the case of CPL and at $60^{\circ} \mathrm{C}$ for CPLG system.

2.3. Physicochemical Characterization. The chitosan-PLLA blends processed by the SSRB technique were subjected to fractional analysis in acidic medium. For the fractionation, a portion of $1.0 \mathrm{~g}$ taken from each sample was immersed into $70 \mathrm{~mL}$ of a $4 \%$ formic acid aqueous solution. After stirring for about 24 hrs at RT, insoluble fractions were collected by filtration, washed on filter with distilled water to $\mathrm{pH} 7$ and dried. The solids contained in the filtrate were precipitated by adding a copious amount of $5 \%$ ammonia aqueous solution, separated from the solutions by centrifugation, washed with water to $\mathrm{pH} \mathrm{7,} \mathrm{and} \mathrm{dried.} \mathrm{The} \mathrm{fractionation} \mathrm{data} \mathrm{obtained}$ for the blends coextruded at various temperatures are listed in Table 1.

Differential scanning calorimetry (DSC) measurements were performed on a Perkin-Elmer DSC-7 instrument 
TABLE 1: Fractionation data for Chitosan-polyester blends obtained at various temperatures. CPL1 blend contains $40 \mathrm{wt} \%$ of chitosan. CPLG and CPL2 blends contain $60 \mathrm{wt} \%$ of chitosan.

\begin{tabular}{|c|c|c|c|c|c|}
\hline Sample code $\mathrm{a}^{\mathrm{a}}$ & $\begin{array}{l}\text { Weight of the } \\
\text { sample (g) }\end{array}$ & $\begin{array}{l}\text { Insoluble in } 4 \% \mathrm{HCOOH} \text { fraction } \\
\text { (enriched with PLLA) }(\mathrm{g} / \mathrm{wt} \%)\end{array}$ & $\begin{array}{c}\text { Soluble fraction }{ }^{\mathrm{b}} \\
\text { (enriched with chitosan) } \\
(\mathrm{g} / \mathrm{wt} \%)\end{array}$ & $\begin{array}{l}\sum \text { weight of } \\
\text { fractions }(g)\end{array}$ & Loss (wt $\%)$ \\
\hline CPLG (60) & 1.7030 & $0.5097 / 29.9$ & $1.0414 / 61.2$ & 1.5511 & 8.9 \\
\hline CPL1 (50) & 0.7368 & $0.5073 / 68.9$ & $0.1996 / 27.1$ & 0.7069 & 4.0 \\
\hline CPL1 (100) & 0.9754 & $0.6074 / 62.3$ & $0.3340 / 34.2$ & 0.9414 & 3.5 \\
\hline CPL2 (100) & 1.0647 & $0.4571 / 42.9$ & $0.5828 / 54.7$ & 1.0399 & 2.3 \\
\hline CPL1 (110) & 1.1824 & $0.7715 / 65.3$ & $0.3609 / 30.5$ & 1.1324 & 4.2 \\
\hline CPL1 (130) & 0.8293 & $0.4985 / 60.1$ & $0.2314 / 27.9$ & 0.7299 & 12.0 \\
\hline CPL2 (130) & 1.0644 & $0.4565 / 42.9$ & $0.5630 / 52.9$ & 1.0195 & 4.2 \\
\hline CPL2 (150) & 1.0478 & $0.4487 / 42.8$ & $0.5572 / 53.2$ & 1.0059 & 4.0 \\
\hline
\end{tabular}

${ }^{a}$ In brackets the temperature of processing of a corresponding mix in extruder is specified.

${ }^{b}$ Weight of precipitated with $5 \% \mathrm{NH}_{4} \mathrm{OH}$ fraction.

calibrated by indium and zinc melting standards. Samples for DSC experiments (weight ca. 5-8 mg) were encapsulated in standard Perkin-Elmer aluminum pans. Dry nitrogen gas was let through the DSC cells at a flow rate of 30 $40 \mathrm{~mL} / \mathrm{min}$, and the measurements were performed over the temperature range of $0-200^{\circ} \mathrm{C}$ at a standard heating rate of $10^{\circ} \mathrm{C} \mathrm{m^{-1 }}$, but various cooling rates were deliberately chosen in the range of $1-200^{\circ} \mathrm{C} \mathrm{min}^{-1}$. The DSC data have been processed with the aid of NETZSCH Proteus Thermal Analysis (V.4.8.4) and NETZSCH Peak Separation (V.2006) programs.

Wide-angle X-ray diffraction patterns were acquired using a Bruker D8 diffractometer operating in a transmission mode in the range of scattering angles $2 \theta=5^{\circ}-100^{\circ}(\mathrm{CuK} \alpha$ radiation). The test samples were represented by melt cast films of $\sim 100 \mu \mathrm{m}$ thickness prepared by press forming at $180^{\circ} \mathrm{C}$.

A Shimadzu AGS-10 universal testing machine was used to measure mechanical characteristics of the films.

Microstructure of the film samples was imagined using a Philips EM-301 SEM. A platinum/carbon replica method was used to prepare the test samples.

Dynamic mechanical analysis (DMA) experiments have been carried out using an automated laboratory-built DMA stand permitting to measure relaxation characteristics of the samples. The measurements were run over the temperature range from -180 to $500^{\circ} \mathrm{C}$ in a resonance vibration (112$190 \mathrm{~Hz}$ ) mode.

Solubility characteristics of the polymer blends were assessed by dynamic light scattering using a Zetatrac particle size analyzer (Microtrac, Inc., USA) with the aid of Microtrac application software program (V 10.5.3). The polymer solutions $(2 \mathrm{mg} / \mathrm{mL})$ were prepared under magnetic stirrer agitation for $2 \mathrm{hrs}$ at RT. The hydrodynamic sizes of the chitosan aggregates generated in organic solvents were determined either with or without preceding sonication. Ultrasound treatment was carried out by using laboratory ultrasonic disperser CUD-500 (Criamid, Russia). The probe tip (diameter $13 \mathrm{~mm}$ ) was immersed in the processing solution down to $\sim 1.5 \mathrm{~cm}$ above the bottom of the sonoreactor vessel. The sonication was run at a fixed wavelength of $20 \mathrm{kHz}$ for $30 \mathrm{sec}$.

${ }^{1} \mathrm{H}-\mathrm{NMR}$ analysis of the polymer solutions $(30 \mathrm{mg} / \mathrm{mL})$ was carried out in $\mathrm{CDCl}_{3}$. The polymers were dissolved at $45^{\circ} \mathrm{C}$ for $22.5 \mathrm{hrs} .{ }^{1} \mathrm{H}-\mathrm{NMR}$ spectra were recorded at $25^{\circ} \mathrm{C}$ with a Bruker AM400 instrument operating at $400.13 \mathrm{MHz}$ by employing TMS as an internal reference. The obtained ${ }^{1} \mathrm{H}-\mathrm{NMR}$ spectra were analyzed with Mnova software.

\section{Results and Discussion}

3.1. Dissolution Study. For the purposes of characterization and processing, we have investigated solubility of the blends in chloroform, the solvent being commonly used for polyester analysis. No one of the samples gave an optically transparent solution in the chlorinated solvent after dissolution for $2 \mathrm{hrs}$ at RT. The solutions in mixed solvent $\left(\mathrm{CH}_{2} \mathrm{Cl}_{2} / \mathrm{DMSO}\right.$ of $\left.9 / 1 \mathrm{v} / \mathrm{v}\right)$ in general seemed to be slightly less turbid. In fact, the both solvents gave only stable dispersions at all the concentrations tested. It may be noted that, in according with the results of visual assessment, the light scattering intensity of the polymer dispersions in the mixed solvent was significantly lower than that in the chloroform. We could thus anticipate that an increase of the solvent polarity significantly enhanced the amphiphilic blend dispersability.

In order to facilitate distribution of the aggregates of modified chitosan in organic solvent medium, an ultrasonic (US) treatment of the polymer dispersions has been performed before comparing their particle sizes derived from the DLS signal. As shown in Figure 1, DLS data indicate the formation of colloidal-scale systems of the CPL1 $\left(130^{\circ} \mathrm{C}\right)$ and CPLG blends prepared at elevated temperatures close to $T_{g}$ of the amorphous polymer and the melting point of the crystalline PLLA. The employed conditions of blending result in bimodal size distribution of chitosan-PLLA blend in $\mathrm{CHCl}_{3}$.

The data presented in Figure 1 demonstrate that US treatment strongly affects diameter of the aggregates unlike that in the mixed solvent. In the latter case, the system is more 


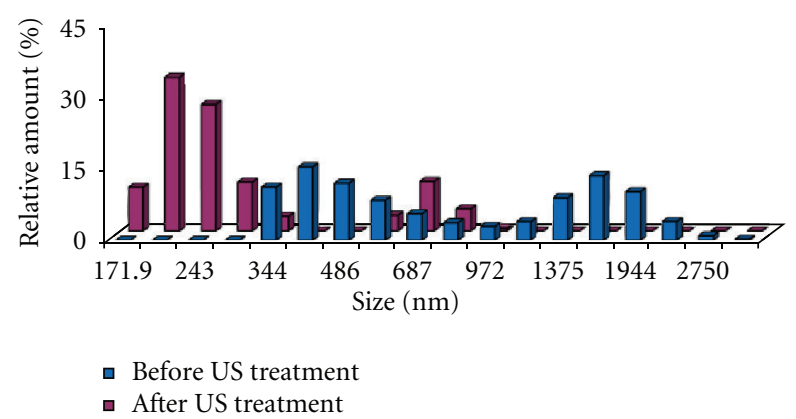

(a)

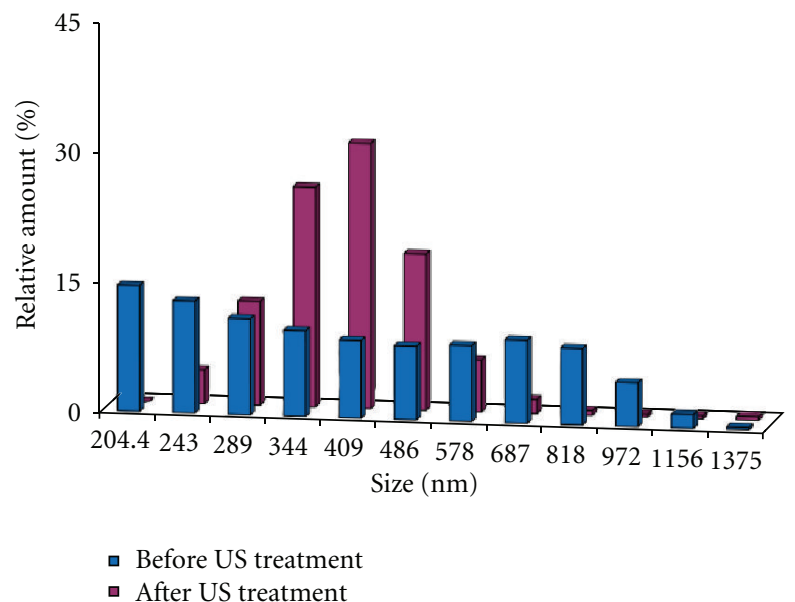

(c)

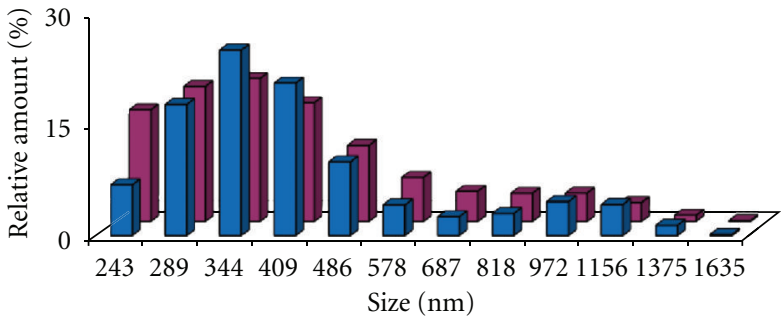

- Before US treatment

- After US treatment

(b)

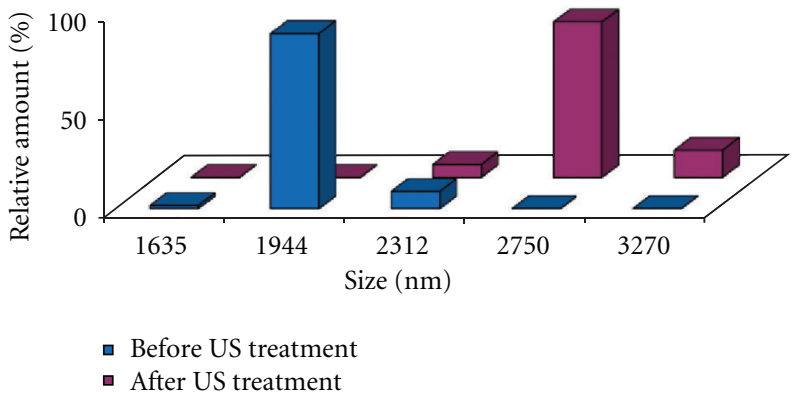

(d)

Figure 1: Number-weighted size distribution in the blend solutions before and after sonication: $\mathrm{CPL} 1 / 130^{\circ} \mathrm{C}$ in $\mathrm{CHCl} 3(\mathrm{a}), \mathrm{CPL} / 130^{\circ} \mathrm{C}$ in mixed solvent $\left(\mathrm{CH}_{2} \mathrm{Cl}_{2} / \mathrm{DMSO}\right.$ of $\left.9 / 1 \mathrm{v} / \mathrm{v}\right)(\mathrm{b}), \mathrm{CPLG} / 60^{\circ} \mathrm{C}(\mathrm{c})$, and $\mathrm{CPL} 2 / 100^{\circ} \mathrm{C}$ (d) both in $\mathrm{CHCl}_{3}$.

uniform regardless of sonication (Figure 1(b)). Figures 1(c) and $1(\mathrm{~d})$ show that chitosan-polyester dispersions containing more chitosan (60 $\mathrm{wt} \%)$ possess a tendency to aggregation in the course of US treatment. However, a mean diameter of the formed CPLG particles is substantially lower $(\sim 360 \mathrm{~nm})$ as compared to all prepared at the same ratio of chitosan to PLLA CPL2 samples which have a mean size of the dispersed phase well above $1 \mu \mathrm{m}$ (Figure 1(d)).

This dissolution study has shown that these polymeric materials exhibit an amphiphilic behavior with propensity to disperse in organic solvents. Obviously, this property is totally different from that would be observed for a physical mixture of the two parent materials, that is, the neat chitosan and polyester. Indeed, whilst the latter would dissolve instantaneously in the selected solvents, the former (polysaccharide) would never dissociate in these solvents at all. It is also worth to mention that the process conditions clearly affect the ability of the composition to form nanoscale dispersion.

Taking into account both the ratio of the components in initial mixture and temperature of the blending, the CPLG blend appears to be more dispersible in organic solvents material as compared to all CPL samples. This property correlates with the differences in crystallinity and average $M_{w}$ of the parent polyesters.
In order to verify the solubility level of the obtained polymeric material sequences in organic medium, we also determined their ${ }^{1} \mathrm{H}-\mathrm{NMR}$ signals in $\mathrm{CDCl}_{3}$. It has been revealed that main proton peaks in NMR spectra correspond mainly to the signals coming from the polyester sequences (Figure 2). The proton peaks in the spectra of both chitosanPLLA and chitosan-PLGA blends are not resolved so well as in the spectra typically observed for homopolyesters. Thus, the NMR data confirm that the polysaccharide moieties are mainly internalized within a core of the micelle-like aggregates generated within organic solvent, and therefore they are not visible in this nuclear spectrometry mode.

3.2. Differential Scanning Calorimetry Measurements. The DSC melting endotherms recorded during the first heating run for neat PLLA polyester and corresponding CPL1 blends prepared by extrusion at various temperatures are shown overlaid in Figure 3(a). In the first heating run, neat PLLA has demonstrated a glass transition at $61.2^{\circ} \mathrm{C}$ with $\Delta C p\left(T_{g}\right)=0.330 \mathrm{Jg}^{-1}$, immediately followed by a cold crystallization exothermic peak, and the melting peak at $T_{m}=165.5^{\circ} \mathrm{C}$ with $\Delta H=40.6 \mathrm{Jg}^{-1}$.

It should be noted that chitosan does not reveal sharp endothermic transitions in the temperature region of 130 $190^{\circ} \mathrm{C}$, and hence all the endotherms shown in Figure 3(a) 


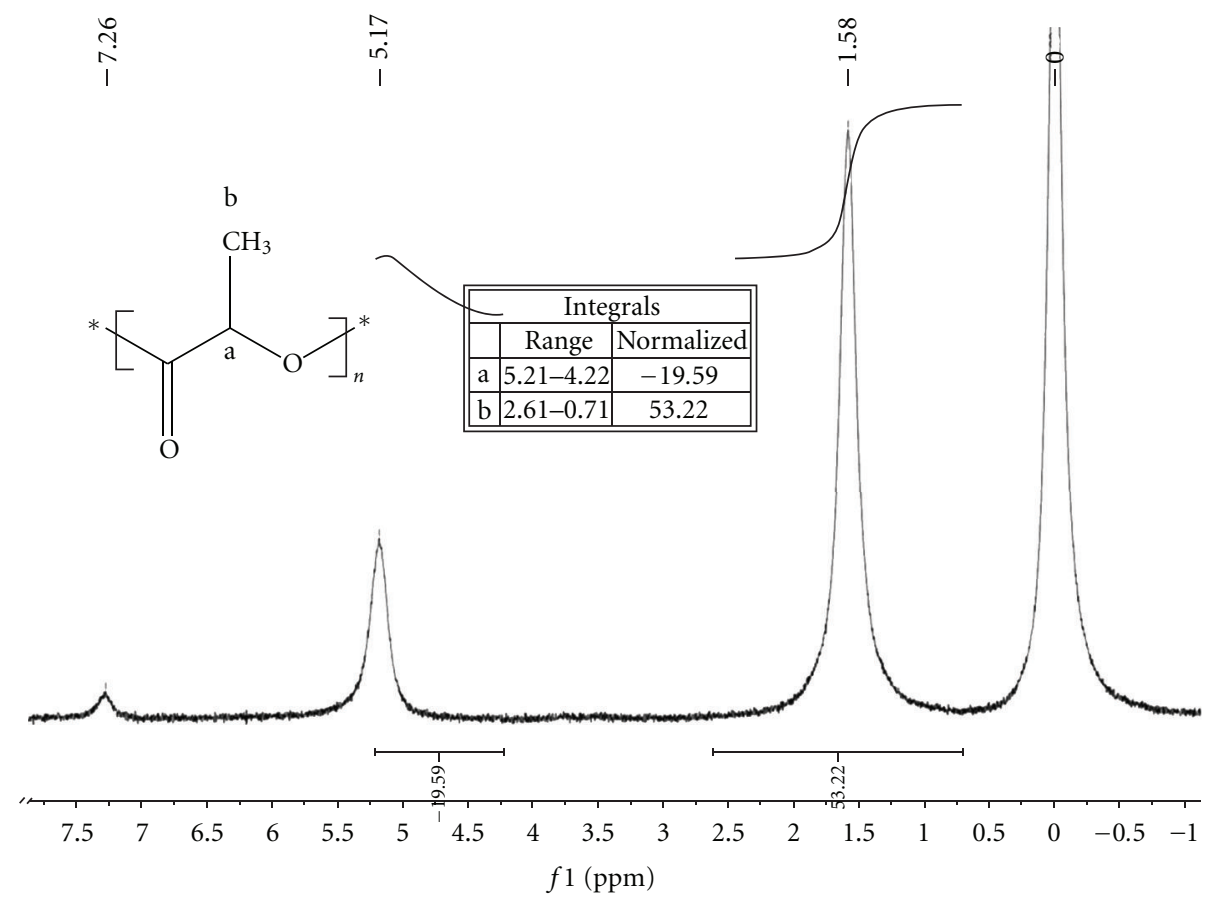

(a)

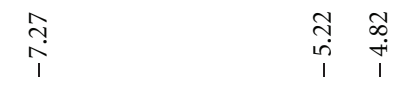

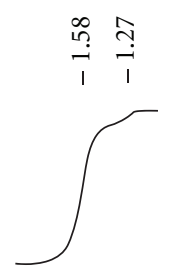<smiles>CC(=O)C(C)COC(=O)COC(C)C</smiles>
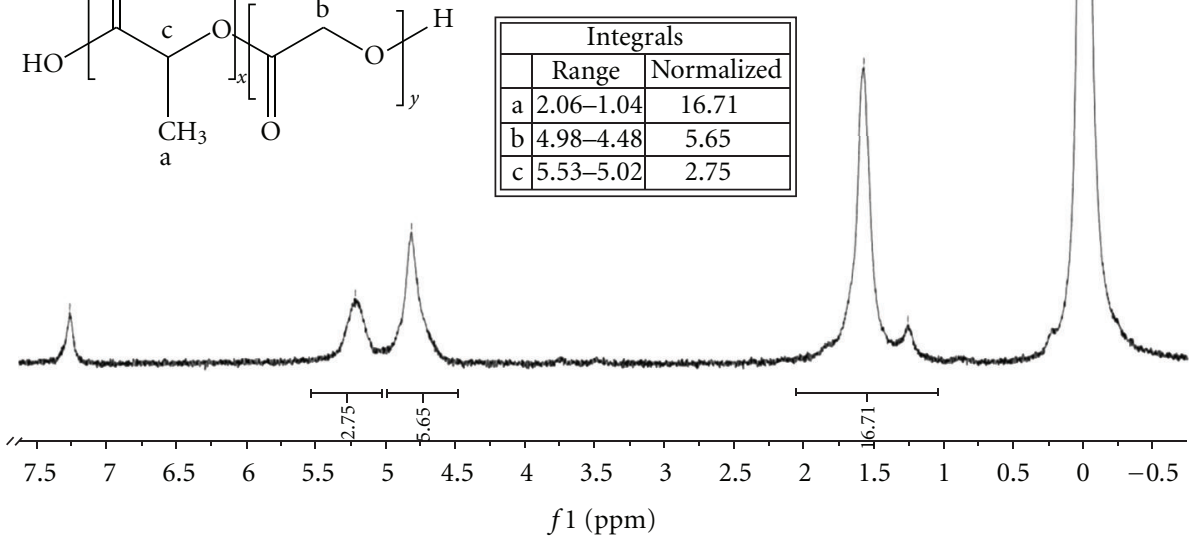

(b)

FIgure 2: ${ }^{1} \mathrm{H}-\mathrm{NMR}$ spectra of $\mathrm{CPL} 1 / 130^{\circ} \mathrm{C}$ (a) and CPLG (b) blends in $\mathrm{CDCl}_{3}$.

originate from PLLA melting. It may be seen that, due to chitosan presence, the PLLA melting endotherms in the compositions DSC curves (cf. curves 2 and 3 ) acquire clear multiphase structure.

Noteworthy, DSC melting endotherm of neat PLLA itself usually consists of two overlapping peaks that can be separated with the aid of curve-fitting routines (Figure 3(c)).
These peaks indicate to coexistence of at least two crystal phases differing in terms of their thermodynamical stability in the parent polymer. Coexistence of these more or less discrete phases in PLLA is commonly related to thermal prehistory of the sample.

Extrusion of PLLA in the presence of chitosan, however, results in preparation of the blends whose melting 


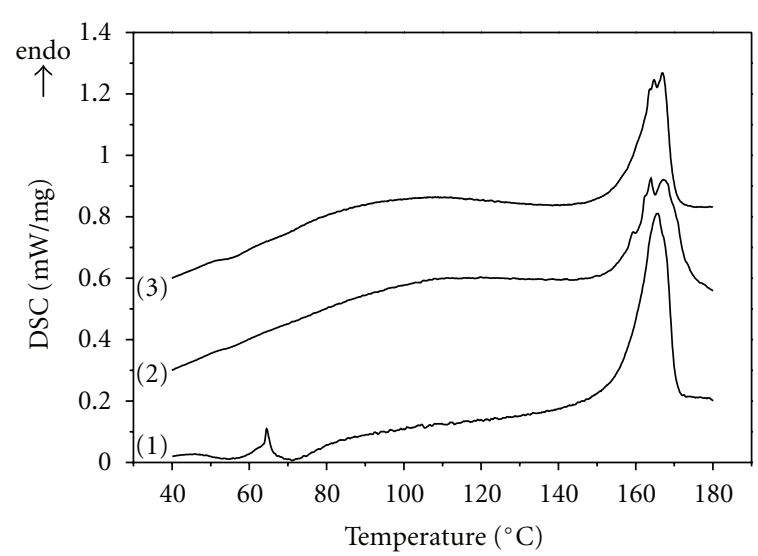

(a)

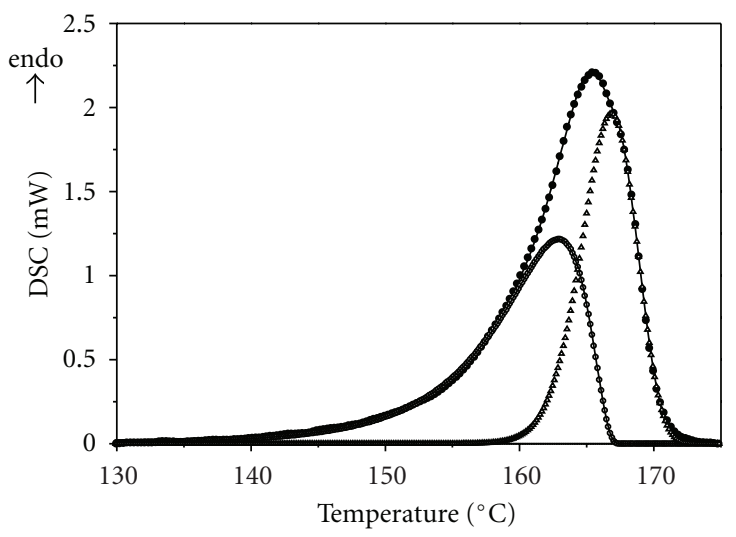

(c)

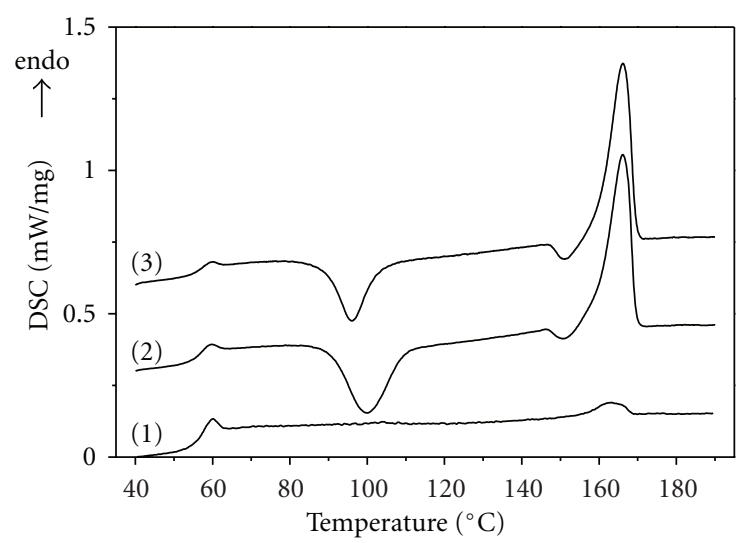

(b)

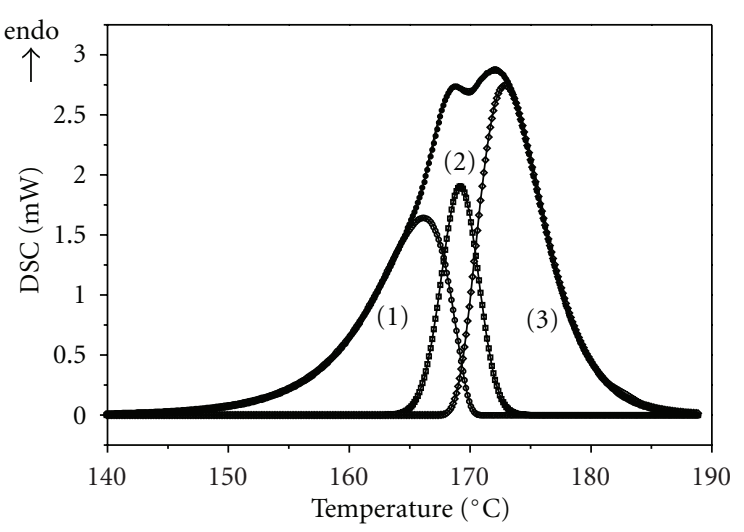

(d)

Figure 3: (a) and (b): First heating run (a) and second heating run (b) DSC curves for neat PLLA (1), CPL1/50 ${ }^{\circ} \mathrm{C}(2)$, and $\mathrm{CPL} 1 / 130^{\circ} \mathrm{C}$ (3) samples. DSC thermograms are offset along the ordinate for clarity. (c) and (d): Curve fitting and separation of the complex melting endotherm for neat PLLA (c) and for CPL $/ 50^{\circ} \mathrm{C}$ sample (d). Partial contributions of the phases into the total endotherm constitute 53.8 and 46.2 relative\% (c); 35.5 (1), 17.9 (2) and 46.6 (3) relative\% (d) correspondingly.

endotherms clearly differ from those characteristics for neat PLLA (Figure 3(a)). Figure 3(d), for instance, shows that the blend endotherm is considerably more complex as compared to that of neat PLLA and comprises complementary peaks belonging to newly formed phases. The latter obviously indicates occurrence of specific interaction of polylactide chains with chitosan moieties and emergence of new PLLA crystallite polymorphs in the presence of chitosan. It is of interest also that the changes in temperature of the extruder treatment have resulted in variations in the phase content of the PLLA endotherm.

For all CPL1 blends melted in DSC and then cooled at $10^{\circ} \mathrm{C} \mathrm{min}^{-1}$ rate, the strong exothermic peaks have been observed during the second DSC heating run in the temperature range of $80-120^{\circ} \mathrm{C}$ (Figure 3(b)). These exotherms belong to the so-called "cold crystallization" of PLLA chains. The fact that neat PLLA has not revealed any cold crystallization peaks at the same conditions infers that chitosan exerts clear nucleating effect on PLLA. This observation is similar to that one reported by Correlo et al. [14] who melt-processed chitosan and PLA at $50 \mathrm{wt} \%$ of chitosan. In that case the PLA cold crystallization in the presence of chitosan was observed in the first heating run. They also render chitosan to be a nucleating agent for PLA. The authors noted, however, that differences could be associated with higher decrease of PLA molecular weight during blend processing.

\subsection{Effect of Copolymer Fraction Formation in the Obtained} Blends. The fractionation data (see Section 2.3, Table 1) clearly indicate to presence of a copolymer fraction in the obtained blends. It can be seen that the blend solutions in aqueous acid contain a fraction which is not precipitated with aqueous ammonia solution. The ninhydrin assay of the solutions after precipitation showed a presence of $\mathrm{NH}_{2}$ groups coming from the modified chitosan in this fraction. It was revealed also that the enriched with chitosan CPL2 samples are well swelling and partially soluble in neutral aqueous medium. According to DSC data, the thermodynamic stability and homogeneity of the crystalline phase as well as the degree of crystallinity (the heat of fusion) of PLLA markedly decrease in CPL2 compositions. The melting peaks have been observed for all CPL2 blends in the temperature range of $155-159^{\circ} \mathrm{C}$ with $\Delta H$ about $20 \mathrm{Jg}^{-1}$ that is considerably lower than those characteristics for neat 


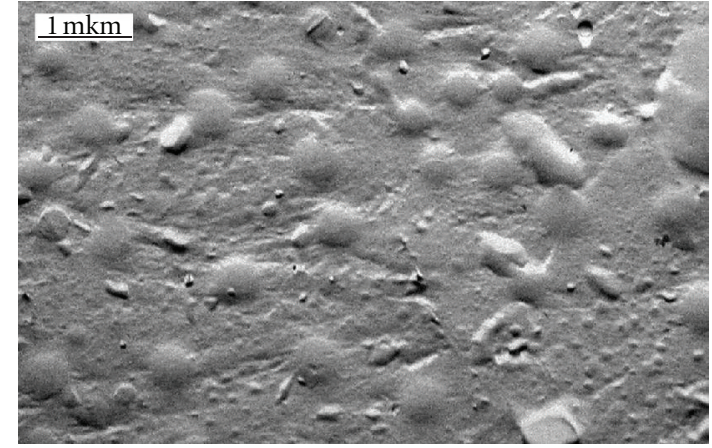

(a)

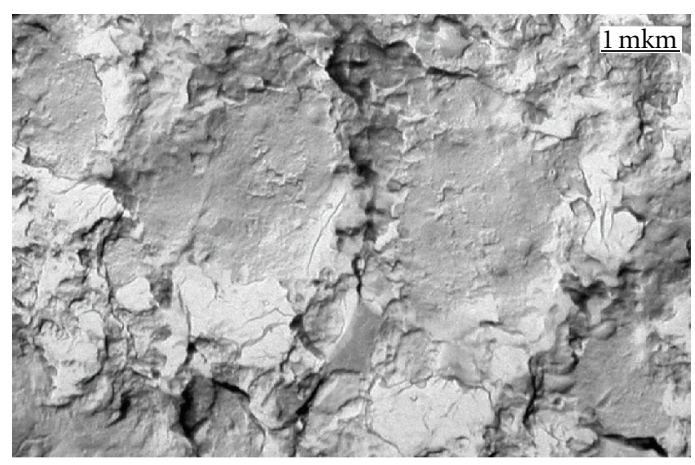

(b)

FIgURE 4: SEM micrographs (magnification $\times 1000$ ) of quenched (a) and annealed (b) hot-pressed films of the $\mathrm{CPL} 1 / 130^{\circ} \mathrm{C}$ blend.

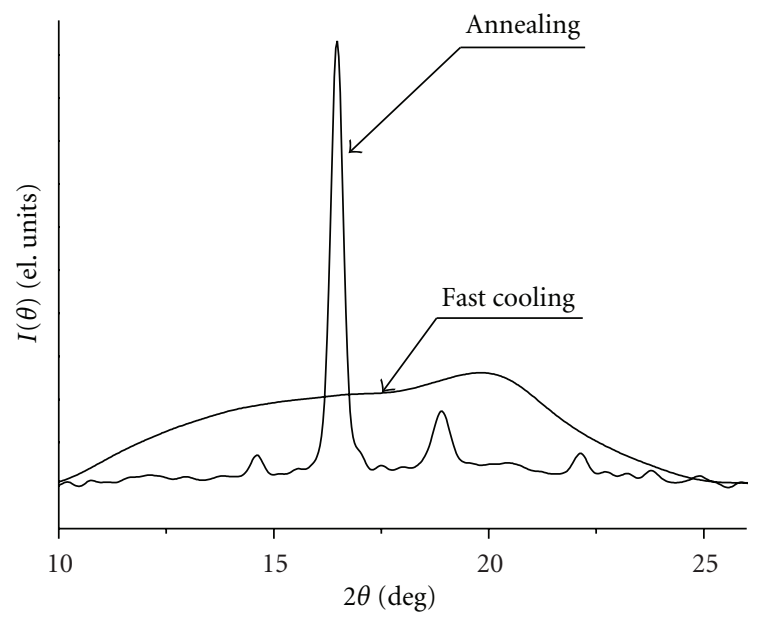

Figure 5: X-ray diffractograms for the $\mathrm{CPL} 1 / 130^{\circ} \mathrm{C}$ films after annealing and fast cooling.

PLLA. We suppose that a high content of the "mechanically hard" chitosan as a component with rather stiff chains assists degradation of PLLA during the SSRB procedure. An increase in rigidity of the CPL2 blends under coextrusion affects the polyester chain length and therefore solubility and thermal properties of the samples.

FTIR spectra of the films cast from stable dispersions in chloroform (prepared by soaking the samples in chloroform at RT for two weeks) contained absorption bands characteristic for both PLLA and chitosan (not shown data). It should be noted that the chitosan content in these stable dispersions based on the CPLG and CPL1 blends prepared at temperatures above $100^{\circ} \mathrm{C}$ was almost the same. With the aid of DLS measurements before and after US treatment it has been shown that the mean particle size of the stable dispersed phase could be changed from $1 \mu \mathrm{m}$ down to $300-500 \mathrm{~nm}$ completely reversibly. Such behavior is quite different from typical behavior of the colloidal systems.

Since unmodified chitosan is not able to dissolve or to swell either in organic solvents or in water at neutral $\mathrm{pH}$ values, the observed amphiphilic behavior of thus prepared chitosan-polyester blends taken together with the fractionation, DLS and FTIR results should infer that coextrusion of the blends resulted to a grafting of the polyester moieties onto chitosan chains. The grafting can be a result of $\mathrm{N}$-acylation of chitosan amino groups by polyester terminal carboxyl groups either as reactions of aminolysis or alcoholysis of the ester bonds of PLLA by chitosan functional groups (Scheme). Moreover, as it has been shown in our previous studies $[21,22]$, ionic interaction between chitosan amino groups and carboxylic groups provides the better intermixing of the components under shear deformation conditions.

3.4. Structural, Relaxation, and Mechanical Properties of the Melt Cast Films. The data presented above does not permit to determine, however, in what crystal form PLLA is present in the blends and whether it forms a separate phase in the blend compositions. In order to evaluate structural and relaxation properties of the obtained materials, SEM, WAXD, and DMA analyses were performed. The melt cast films of the coextruded CPL blends were used as the test samples. The mechanical properties of the films were measured as well.

The morphology of annealed and quenched hot-pressed films of CPL1 blend coextruded at $130^{\circ} \mathrm{C}$ was analyzed with SEM. In order to expose supramolecular structure of the samples, oxygen plasma-treatment technique was applied for etching the film surface. It can be seen in Figure 4 that both types of the obtained blend membranes possess a clear twophase structure. In the study of quenched films (Figure 4(a)), a number of chitosan spherical aggregates $(\sim 200-400 \mathrm{~nm}$ in diameter) evenly dispersed over a continuous PLLA matrix have been observed. In the case of the films prepared by slow cooling of the melt, PLLA crystallizes, and the film structure became microheterogeneous (Figure 4(b)).

Figure 5 shows WAXD analysis for slowly annealed and rapidly quenched hot-pressed films of the CPL1 blend coextruded at $130^{\circ} \mathrm{C}$. Thus prepared films were compared in terms of their morphology with films of a model mixture of the same composition (chitosan/PLLA $=40 / 60 \%$ by weight) prepared with the aid of microcompounder at $190^{\circ} \mathrm{C}$ and then cast from the melt. The size of chitosan particles in the latter case remained unchanged (about $100 \mu \mathrm{m}$ ), and the samples visually appeared to be heterogeneous. The films 


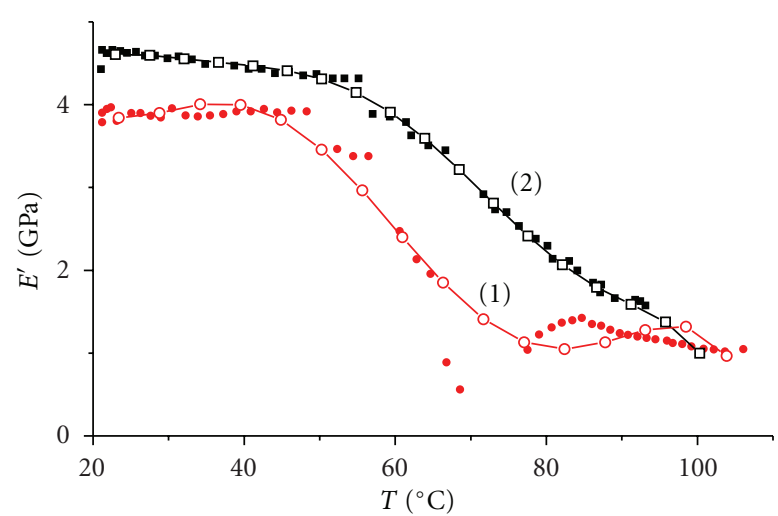

(a)

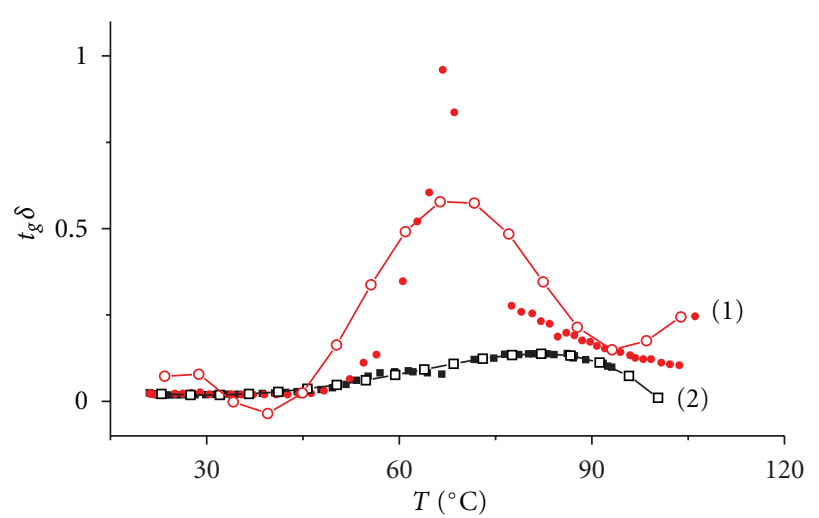

(b)

Figure 6: Temperature dependence of storage modulus $E^{\prime}$ (a) and the mechanical-loss tangent (b) for amorphous (1) and crystalline (2) films of the $\mathrm{CPL} 1 / 130^{\circ} \mathrm{C}$ sample.

TABle 2: Tensile properties of the films of neat PLLA and chitosanPLLA blends treated in extruder at various temperatures.

\begin{tabular}{lccc}
\hline Sample & $\begin{array}{c}\text { Tensile } \\
\text { strength } \\
(\mathrm{MPa})\end{array}$ & $\begin{array}{c}\text { Strain at } \\
\text { tensile } \\
\text { strength }(\%)\end{array}$ & $\begin{array}{c}\text { Modulus of } \\
\text { elasticity } \\
(\mathrm{GPa})\end{array}$ \\
\hline PLLA & 48.0 & 2.4 & 2.3 \\
CPL1 (50) fast cooling & 51.0 & 2.0 & 2.9 \\
CPL1 (100) fast cooling & 32.0 & 1.5 & 2.3 \\
CPL1 (130) fast cooling & 18.3 & 0.7 & 2.7 \\
CPL1 (130) annealing & 21.3 & 0.7 & 3.0 \\
CPL2 (100) fast cooling & 40.0 & 1.6 & 2.6 \\
\hline
\end{tabular}

prepared in this model experiment were highly brittle and unsuitable to evaluate their mechanical properties.

In order to evaluate relaxation properties of the samples, the films of the solid-state extruded blends have been subjected to dynamic mechanical analysis. According to the DMA data, fast cooling of the composite films, promoting amorphization of PLLA phases, has resulted to a decrease of the dynamic storage modulus $E^{\prime}$ of the sample (Figure 6(a)). On the temperature dependence of the mechanical-loss tangent for the quenched film (Figure 6(b)), the strong peak at $60^{\circ} \mathrm{C}$ has been observed due to glass transition of PLLA that correlates with DSC data. The sample subjected to annealing demonstrates the same relaxation process but not so clearly manifested. It is corresponding to the region of a maximum decrease of the dynamic storage modulus $E^{\prime}$ of the samples. In both composite films the transition in the state of plastic flow is not completed. It is obvious that mechanical properties of compositions above $T_{g}$ of PLLA are determined exclusively by the chitosan matrix. Unlike hydrophilic systems based on chitosan, the given system is stable in terms of mechanical properties in the presence of moisture.

The tensile strength, modulus of elasticity, and elongation values of the CPL films are shown in Table 2. The tensile strength of the films of CPL1 blend prepared at low-temperature extrusion $\left(50^{\circ} \mathrm{C}\right)$ is close to the values characteristic for neat PLLA. Surprisingly enough that the similar observation can be made for CPL2 films containing $60 \%$ wt of chitosan in the blends prepared at $100^{\circ} \mathrm{C}$. According to DLS data (see Figure 1(d)), both samples possess rather uniform size distribution of the dispersed phase with a mean size of the particles of $1.7-2 \mu \mathrm{m}$. Obviously, the low-temperature blending allows to minimize mechanical and oxidative degradation of the PLLA and thereby to preserve the mechanical properties of the continuous polyester matrix. When higher coextrusion temperatures were used the tensile strength of the corresponding films was decreased. The reasons can be aminolysis reactions and alcoholysis of the ester bonds of PLLA by chitosan functional groups occurring under the SSRB conditions (see Scheme 1 unit). These reactions lead to grafting polyester moieties onto chitosan chain and formation of uniform structure of the chitosan-polyester materials as it has been confirmed by DLS and SEM results.

3.5. Biomaterials Preparation and Future Prospects. A propensity of the obtained materials to disperse in organic solvents on colloidal level allows obtaining of microfibers with chitosan content of $40 \mathrm{wt} \%$ by electrospinning process [23]. The developed blends were also used for biodegradable microbead manufacturing according to simple oil-in-water emulsion solvent evaporation technique. Viewed under optical microscope, the prepared polymer microparticles have a spherical shape and, therefore, could be promising materials for their further usage for cell cultivation and as microcarriers for wound healing and tissue regeneration. Chitosan-based polymeric matrices are, besides, potentially useful to prepare hybrid organic-inorganic composites. The possibility for chitosan amino groups to interact with the surface layer of nanostructured fillers $[24,25]$ as well as rather good processability of protein substrates under SSRB conditions [26] can be exploited to design materials combining a better structural integrity and flexibility along with improved bioactivity. Such composites have gained 

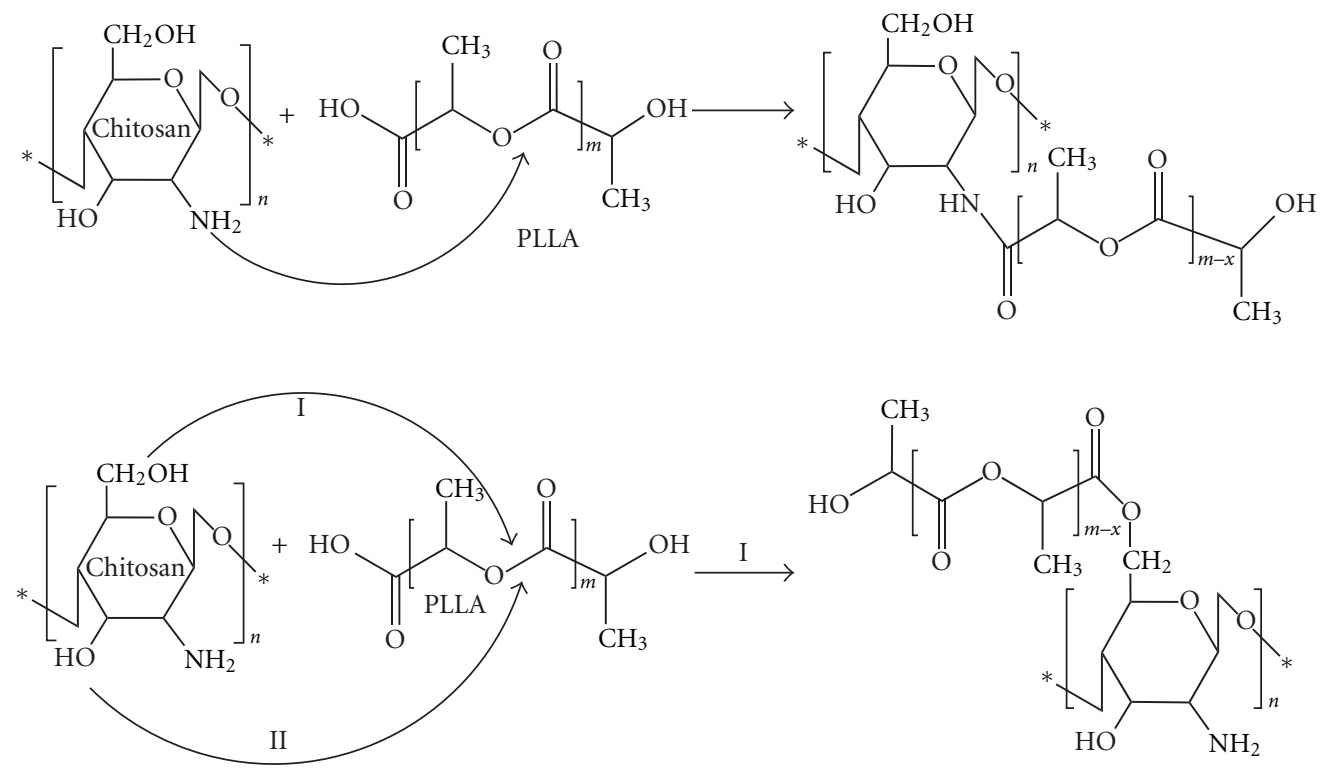

Scheme 1: Aminolysis (1) and alcoholysis (2) reactions of the ester bonds by chitosan functional groups.

increasing interest in the field of tissue engineering which appears as a promising concept for bone reconstruction. This direction is an independent subject of our further studies.

\section{Conclusions}

In this study, we evaluated the possibilities of the solid-state reactive blending as an efficient and ecologically safe method to synthesize chitosan-polyester materials. Accordingly, a number of chitosan-PLLA (PLGA) blends were successfully prepared by coextrusion at various temperatures below the melting points of the crystalline polymers. DSC data indicate that extrusion of PLLA in the presence of chitosan at all temperatures leads to intense interaction between the components. Mechanical activation and effective intermixing of the polymers under shear deformation have resulted in compatibilization of the components followed by grafting of polyester moieties onto chitosan chains at elevated temperatures close to $T_{g}$ of amorphous polymer and the melting point of the crystalline PLLA. It has been found that the employed technique leads to substantial modification of physical-chemical properties of the polysaccharide, in particular its propensity to disperse in organic solvents. According to DLS data, the materials produced at optimal conditions of blending form colloidal chitosan/polyester/chloroform systems with a mean size of the dispersed phase of 200 $400 \mathrm{~nm}$. This propensity of the obtained materials allows manufacturing of microfibers and microbeads with chitosan content up to $60 \mathrm{wt} \%$ for the design of polyester-based biomaterials with improved bioactivity.

The confirmed by SEM uniform structure of hot-pressed films of the obtained blends is responsible for the observed enhancement of their mechanical properties as compared with a model molten system with the same composition.
Tensile strength of the films made of blends processed at low temperature turned out to be similar to that of neat PLLA.

Compared with traditional methods for manufacture of hybrid polysaccharide-based materials, the solid-state reactive blending is a relatively simple, cost effective, and convenient way to improve the composite properties. This approach may be rendered as one of the most promising techniques to design chitosan-polylactide materials which can be extended to a wide range of polysaccharides and polyesters. The avoiding of any catalysts, initiators, as well as organic solvents during processing allows to improve purity of the final composite matrices. The developed polymeric materials are, therefore, potentially useful for various biomedical applications.

\section{Acknowledgments}

The authors express their gratitude to Professor Elena Markvicheva (Shemyakin \& Ovchinnikov Institute of Bioorganic Chemistry of RAS) and Professor Anatoly Chalykh (Institute of Physical Chemistry and Electrochemistry of RAS) for providing sample analyses and fruitful discussions throughout this work. This research was supported by a grant from the Russian Foundation for Basic Research (RFBR, Project no. 10-03-01022-a). Additional support provided by SS-4371.2010.3 is gratefully acknowledged.

\section{References}

[1] D. Garlotta, "A literature review of poly(lactic acid)," Journal of Polymers and the Environment, vol. 9, no. 2, pp. 63-84, 2001.

[2] P. Simamora and W. Chern, "Poly-L-lactic acid: an overview," JDD: Journal of Drugs in Dermatology, vol. 5, no. 5, pp. 436440, 2006. 
[3] L. Lu, S. J. Peter, M. D. Lyman et al., "In vitro and in vivo degradation of porous poly(DL-lactic-co-glycolic acid) foams," Biomaterials, vol. 21, no. 18, pp. 1837-1845, 2000.

[4] Y. P. Jiao and F. Z. Cui, "Surface modification of polyester biomaterials for tissue engineering," Biomedical Materials, vol. 2, no. 4, pp. R24-R37, 2007.

[5] R. A. A. Muzzarelli and C. Muzzarelli, "Chitosan chemistry: relevance to the biomedical sciences," Advances in Polymer Science, vol. 186, pp. 151-209, 2005.

[6] K. C. Gupta and M. N. V. Ravi Kumar, "An overview on chitin and chitosan applications with an emphasis on controlled drug release formulations," Journal of Macromolecular Science, vol. 40, no. 4, pp. 273-308, 2000.

[7] N. M. Breyner, R. C. R. Hell, L. R. P. Carvalho et al., "Effect of a three-dimensional chitosan porous scaffold on the differentiation of mesenchymal stem cells into chondrocytes," Cells Tissues Organs, vol. 191, no. 2, pp. 119-128, 2010.

[8] R. Jin, L. S. Moreira Teixeira, P. J. Dijkstra et al., "Injectable chitosan-based hydrogels for cartilage tissue engineering," Biomaterials, vol. 30, no. 13, pp. 2544-2551, 2009.

[9] J. K. Francis Suh and H. W. T. Matthew, "Application of chitosan-based polysaccharide biomaterials in cartilage tissue engineering: a review," Biomaterials, vol. 21, no. 24, pp. 25892598, 2000.

[10] Y. L. Cui, A. D. Qi, W. G. Liu et al., "Biomimetic surface modification of poly(L-lactic acid) with chitosan and its effects on articular chondrocytes in vitro," Biomaterials, vol. 24, no. 21, pp. 3859-3868, 2003.

[11] N. Bhattarai, H. R. Ramay, S. H. Chou, and M. Zhang, "Chitosan and lactic acid-grafted chitosan nanoparticles as carriers for prolonged drug delivery," International journal of nanomedicine, vol. 1, no. 2, pp. 181-187, 2006.

[12] F. Sébastien, G. Stéphane, A. Copinet, and V. Coma, "Novel biodegradable films made from chitosan and poly(lactic acid) with antifungal properties against mycotoxinogen strains," Carbohydrate Polymers, vol. 65, no. 2, pp. 185-193, 2006.

[13] F. Yao, W. Chena, H. Wang et al., "A study on cytocompatible poly(chitosan-g-L-lactic acid)," Polymer, vol. 44, no. 21, pp. 6435-6441, 2003.

[14] V. M. Correlo, L. F. Boesel, M. Bhattacharya, J. F. Mano, N. M. Neves, and R. L. Reis, "Properties of melt processed chitosan and aliphatic polyester blends," Materials Science and Engineering A, vol. 403, no. 1-2, pp. 57-68, 2005.

[15] A. N. Ozerin, A. N. Zelenetskii, T. A. Akopova et al., RF Patent 2292354, 2007.

[16] A. A. Zharov, "Reactions of solid monomers and polymers under shear deformation and high pressure," in High-Pressure Chemistry and Physics of Polymers, A. L. Kovarskii, Ed., pp. 267-301, CRC Press, London, UK, 1994.

[17] V. A. Zhorin, "Processes accompanying plastic flow under high pressure in polymers and low-molecular substances (review)," Polymer Science Series A, vol. 36, no. 4, pp. 559-579, 1994.

[18] E. V. Prut and A. N. Zelenetskii, "Chemical modification and blending of polymers in an extruder reactor," Russian Chemical Reviews, vol. 70, no. 1, pp. 65-79, 2001.

[19] P. Y. Butyagin, "Forced reactions in inorganic and organic chemistry," Colloid Journal of the Russian Academy of Sciences, vol. 61, no. 5, pp. 537-544, 1999.

[20] S. Z. Rogovina, T. A. Akopova, and G. A. Vikhoreva, "Investigation of properties of chitosan obtained by solid-phase and suspension methods," Journal of Applied Polymer Science, vol. 70, no. 5, pp. 927-933, 1998.

[21] S. Z. Rogovina, G. A. Vikhoreva, T. A. Akopova, and I. N. Gorbacheva, "Investigation of interaction of chitosan with solid organic acids and anhydrides under conditions of shear deformation," Journal of Applied Polymer Science, vol. 76, pp. 616-622, 2000.

[22] T. S. Demina, T. A. Akopova, L. V. Vladimirov et al., "The study of the interaction between chitosan and 2,2bis(hydroxymethyl) propionic acid during solid-phase synthesis," Polymer Science-Series B, vol. 53, no. 5-6, pp. 358-370, 2011.

[23] T. A. Akopova, E. A. Markvicheva, A. N. Zelenetskii, A. N. Ozerin, L. V. Vladimirov, and Y. D. Livney, "Solid-state reactive blending - a promising way to biomedical polymer materials," in Proceedings of the 3rd International Conference Fundamental Basis of Mechanochemical Technologies (FBMT '09), p. 80, Novosibirsk, Russia, 2009.

[24] C. Muzzarelli and R. A. A. Muzzarelli, "Natural and artificial chitosan-inorganic composites," Journal of Inorganic Biochemistry, vol. 92, no. 2, pp. 89-94, 2002.

[25] A. N. Ozerin, N. S. Perov, A. N. Zelenetskii et al., "Hybrid nanocomposites based on graft copolymer of chitosan with poly(vinyl alcohol) and titanium oxide," Nanotechnologies in Russia, vol. 4, no. 5-6, pp. 331-339, 2009.

[26] A. N. Zelenetskii, T. A. Akopova, N. R. Kildeeva, G. A. Vikhoreva, E. S. Obolonkova, and A. A. Zharov, "Immobilization of trypsin on polysaccharides upon intense mechanical treatment," Russian Chemical Bulletin, vol. 52, no. 9, pp. 2073 2077, 2003. 

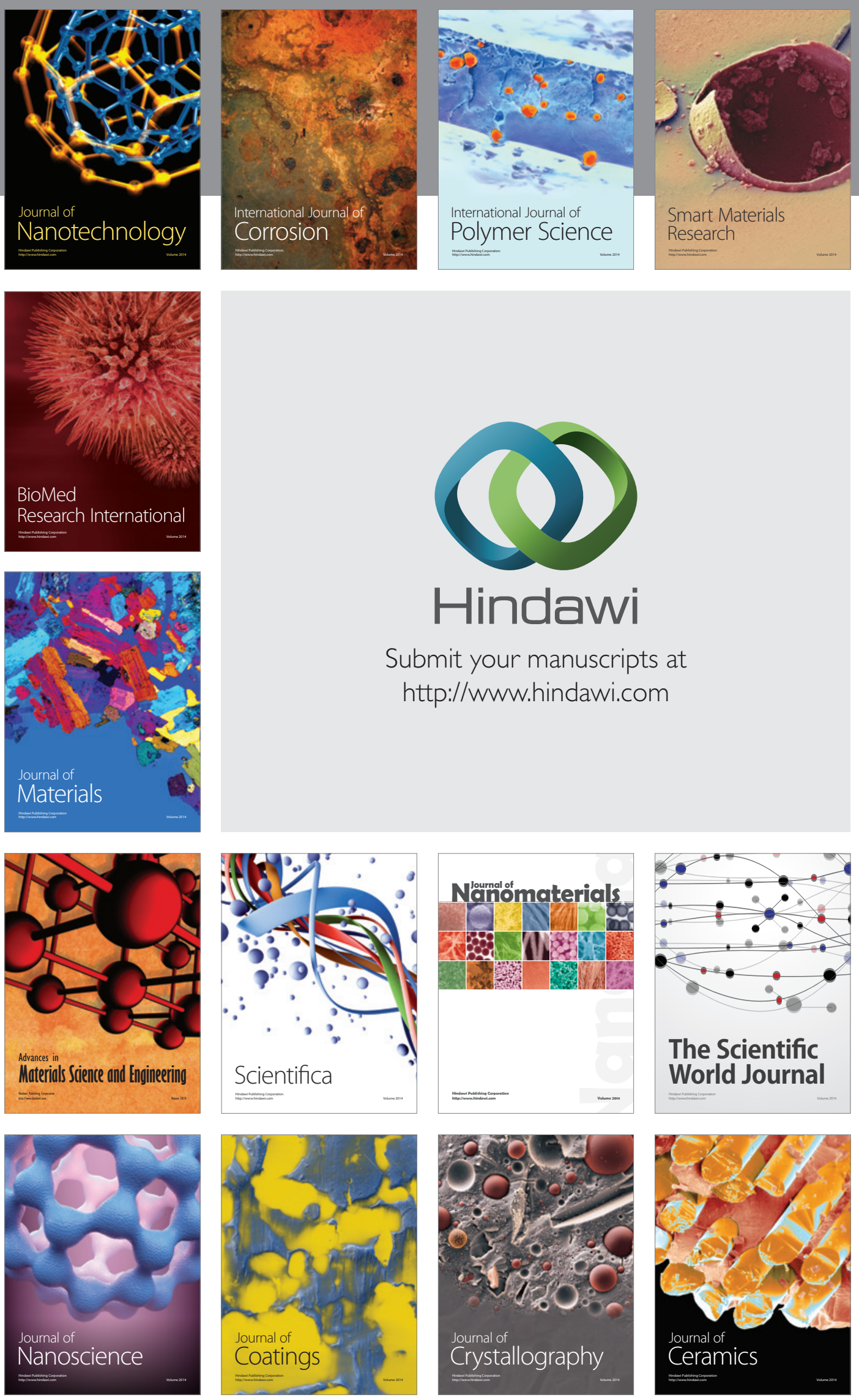

The Scientific World Journal

Submit your manuscripts at

http://www.hindawi.com

\section{World Journal}

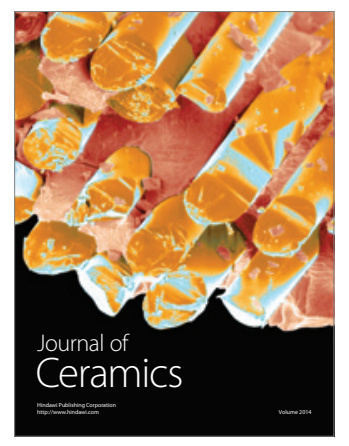

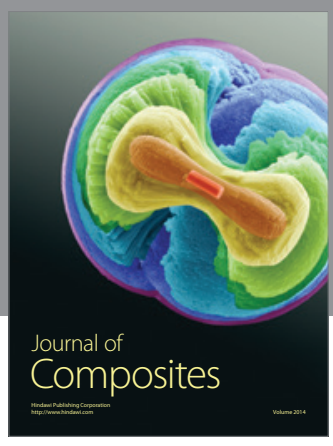
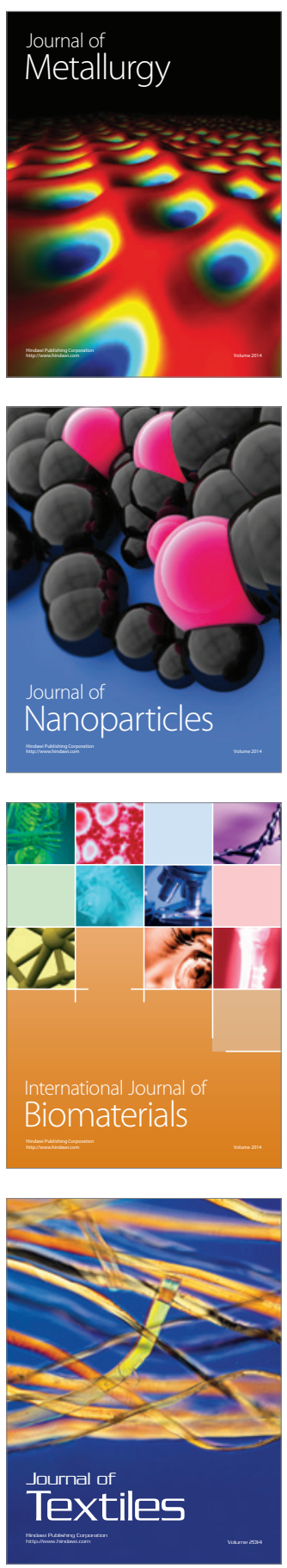